\title{
Fluoxetine-induced alteration of murine gut microbial community structure: Evidence for a microbial endocrinology- based mechanism of action responsible for fluoxetine-induced side effects
}

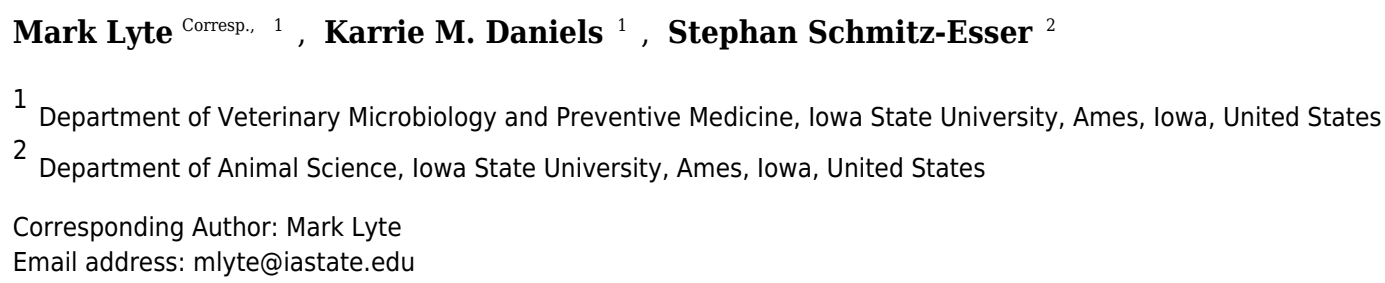

Background. Depression and major depressive disorder affect $25 \%$ of the population. First line treatment utilizing selective serotonin reuptake inhibitors (SSRIs) have met with limited success due to well-recognized negative side effects which include weight gain or loss. This inability to control unwanted side effects often result in patients stopping their antidepressant medications. The mechanisms underlying the failure of SSRIs are incompletely understood. Methods. Male CF-1 mice ( 5 weeks of age, $\mathrm{N}=10$ per group) were per orally administered fluoxetine (20 mg per kg body weight) or diluent daily for 29 days. During this time fecal specimens were collected at three defined time points $(0,15$ and 29 days). At the conclusion of the 29-day dosing regimen, animals were subjected to two behavioral assessments. For bacterial identification of the microbiota, 16S rRNA gene sequencing was performed on $60 \mathrm{fecal}$ specimens ( 3 specimens per mouse time course, $\mathrm{N}=20$ mice) using Illumina MiSeq. Analysis of community sequence data was done using mothur and LEfSe bioinformatic software packages. Results. Daily per oral administration of fluoxetine for 29 days to male mice resulted in a significant, time dependent, alteration in microbial communities accompanying changes in body weight. The calculated species richness and diversity indicators of the murine fecal microbial communities were inconsistent and not significantly different within between the groups. Among the phylotypes decreased in abundance due to fluoxetine administration were Lactobacillus johnsonii and Bacteroidales S24-7 which belong to phyla associated with regulation of body mass. The observed changes in body weight due to fluoxetine administration mimicked the dramatic shifts in weight gain/loss that has been observed in humans. Further, at the conclusion of the 29-day dosing regimen fluoxetine-dosed animals evidenced a mild anxiogenic-like behavior. Discussion. We report that the most widely used antidepressant, fluoxetine, which is an SSRI-type drug, results in the selective 
depletion of gut microbiota, specifically the Lactobacilli which are involved in the regulation of body weight. Concomitantly, fluoxetine administration increases the abundance of phylotypes related to dysbiosis. Since Lactobacilli have been previously shown to possess a known biogenic amine transporter that regulates the uptake of fluoxetine, it is proposed that a microbial endocrinology-based mechanistic pathway is responsible for the ability of SSRIs to selectively negatively impact beneficial microbiota. The results of this study therefore suggest that the negative clinical side effects due to fluoxetine administration may be due to alterations in gut microbiota. Further, the data also suggests that supplementation of bacterial genera directly affected by fluoxetine administration may prove useful in ameliorating some of the well-known side effects of chronic fluoxetine administration such as weight alterations. 
1 Fluoxetine-induced alteration of murine gut microbial community structure: Evidence for

2 a microbial endocrinology-based mechanism of action responsible for fluoxetine-induced

3

4

5

$6{ }^{1}$ Department of Veterinary Microbiology and Preventive Medicine, Iowa State University, Ames,

7 IA 50011-1134, USA

8

$9 \quad{ }^{2}$ Department of Animal Science, Iowa State University, Ames, IA 50011-3611, USA

10

11 Corresponding Author:

12 Mark Lyte ${ }^{1}$

131800 Christensen Drive, Ames, IA 50011-1134, USA

14 Email address: mlyte@iastate.edu (ML) side effects

Mark Lyte $^{1 *}$, Karrie M. Daniels ${ }^{1}$ and Stephan Schmitz-Esser ${ }^{2}$ 


\section{ABSTRACT}

16 Background. Depression and major depressive disorder affect $25 \%$ of the population. First line

17 treatment utilizing selective serotonin reuptake inhibitors (SSRIs) have met with limited success

18 due to well-recognized negative side effects which include weight gain or loss. This inability to

19 control unwanted side effects often result in patients stopping their antidepressant medications.

20 The mechanisms underlying the failure of SSRIs are incompletely understood.

21 Methods. Male CF-1 mice ( 5 weeks of age, $\mathrm{N}=10$ per group) were per orally administered

22 fluoxetine (20 mg per kg body weight) or diluent daily for 29 days. During this time fecal

23 specimens were collected at three defined time points (0, 15 and 29 days). At the conclusion of

24 the 29-day dosing regimen, animals were subjected to two behavioral assessments. For bacterial

25 identification of the microbiota, 16S rRNA gene sequencing was performed on 60 fecal

26 specimens ( 3 specimens per mouse time course, $\mathrm{N}=20$ mice) using Illumina MiSeq. Analysis of

27 community sequence data was done using mothur and LEfSe bioinformatic software packages.

28 Results. Daily per oral administration of fluoxetine for 29 days to male mice resulted in a

29 significant, time dependent, alteration in microbial communities accompanying changes in body

30 weight. The calculated species richness and diversity indicators of the murine fecal microbial

31 communities were inconsistent and not significantly different within between the groups. Among

32 the phylotypes decreased in abundance due to fluoxetine administration were Lactobacillus

33 johnsonii and Bacteroidales $S 24-7$ which belong to phyla associated with regulation of body

34 mass. The observed changes in body weight due to fluoxetine administration mimicked the

35 dramatic shifts in weight gain/loss that has been observed in humans. Further, at the conclusion 
36 of the 29-day dosing regimen fluoxetine-dosed animals evidenced a mild anxiogenic-like

37 behavior.

38 Discussion. We report that the most widely used antidepressant, fluoxetine, which is an SSRI-

39 type drug, results in the selective depletion of gut microbiota, specifically the Lactobacilli which

40 are involved in the regulation of body weight. Concomitantly, fluoxetine administration

41 increases the abundance of phylotypes related to dysbiosis. Since Lactobacilli have been

42 previously shown to possess a known biogenic amine transporter that regulates the uptake of

43 fluoxetine, it is proposed that a microbial endocrinology-based mechanistic pathway is

44 responsible for the ability of SSRIs to selectively negatively impact beneficial microbiota. The

45 results of this study therefore suggest that the negative clinical side effects due to fluoxetine

46 administration may be due to alterations in gut microbiota. Further, the data also suggests that

47 supplementation of bacterial genera directly affected by fluoxetine administration may prove

48 useful in ameliorating some of the well-known side effects of chronic fluoxetine administration

49 such as weight alterations. 


\section{INTRODUCTION}

51 The failure of drug therapy to adequately treat depression in large segments of the

52 population and the numerous side effects that accompany its chronic administration such as

53 anxiogenic effects and changes in body weight has been well recognized for years in both human

54 (Anderson 1998; Cascade et al. 2010) as well as animal populations (Aggarwal et al. 2016;

55 Bagdy et al. 2001). Antidepressants, notably the selective serotonin reuptake inhibitors,

56 otherwise referred to as SSRIs, are considered the first line of treatment for anxiety and major

57 depressive disorder (Williams et al. 2017). SSRIs, as with the great majority of drugs, are taken

58 per orally. Although the pharmacokinetic analysis of per oral dosing of SSRIs has demonstrated

59 that the majority is absorbed into the host with peak plasma concentration occurring within 4-6

60 hours following, a significant percentage of the administered per oral dose can in fact be

61 recovered in the feces as has been shown in human volunteers (Lemberger et al. 1985). Thus,

62 in addition to the host, the microbiota also represents a biological entity that interacts with any

63 administered drug such as SSRIs. However, a crucial difference is that far less is known

64 concerning the consequences of antipsychotic drug-microbiota interactions than are known for

65 the host. Little, if any, research has been done on the mechanisms governing the effects of

66 antidepressants on the microbiota and whether the failure and side effects of antidepressant

67 therapy may be due to altered drug-induced microbiota. That it is entirely plausible that the

68 microbiota composition, and in turn function, can be influenced by oral antipsychotic drugs can

69 be seen in published evidence that has shown that among the large diversity of non-antibiotic

70 drugs administered within the general population, antipsychotic drugs had potent effects on fecal

71 microbial diversity (Maier et al. 2018). Additionally, a recent study analyzed the effect of 
72 fluoxetine on gut microbiota in rats (Cussotto et al. 2018), but so far, the effect of fluoxetine on

73 microbiota composition remains largely unknown.

74 In proposing a direct microbial effect of SSRIs, we have employed a microbial

75 endocrinology-based theoretical framework as it has as its basis the concept of shared

76 neurochemistry between microbes and host enabling bi-directional communication (Lyte 2014).

77 This approach has led to the recent discovery that selective bacterial genera within the gut

78 microbiota, such as the Lactobacilli, possess the same two biogenic amine transports which are

79 found in mammalian neuronal cells and are intimately involved in the therapeutic action of

80 SSRIs (Lyte \& Brown 2018). The plasma membrane monoamine transporter (PMAT)- and the

81 organic cation transporter (OCT)-like were shown to be present on Lactobacillus salivarius, but

82 not L. rhamnosus, and display similar activity in regard to reuptake inhibitors such as the SSRI

83 fluoxetine as is observed in mammalian neuronal cell cultures (Lyte \& Brown 2018).

84 As such, we hypothesized that per oral, chronic administration of fluoxetine could

85 influence the diversity of the gut microbiota through direct microbial endocrinology-based

86 interactions. This interaction would, by definition, be specific to only those bacterial genera

87 which exhibit cell-based mechanisms, such as biogenic amine transporters, with which to interact

88 with fluoxetine. This differs from previous reports which extend back decades which have

89 reported that a number of psychoactive drugs can negatively influence bacterial viability and

90 even suggested as an adjunct to therapy of a number of infectious diseases as the addition of an

91 SSRI could lower the minimum inhibitory concentration of an antimicrobial with certain multi-

92 drug resistant pathogens (Munoz-Bellido et al. 1996; Munoz-Bellido et al. 2000). The present

93 study was designed to examine if a genus-specific effect of fluoxetine administration could be

94 shown. If so, it then would become plausible to suggest that the ability of fluoxetine to influence 
95 host behavior as well as engender unwanted side effects could be due to direct microbial 96 endocrinology-based effects within the host gut microbiota. 


\section{MATERIALS AND METHODS}

98 Animals. Twenty male CF-1 mice at 5 weeks of age (Charles River Laboratories, Wilmington,

99 MA, USA) were randomized upon receipt and then housed at a density of 2 per cage

100 (McCafferty et al. 2013). All experimental procedures were approved by the Iowa State

101 University Institutional Animal Care and Use Committee, protocol \#1-17-8420-M. Mice were

102 weighed every other day in order to ensure that the correct mg per kg dosage was being

103 administered. As shown (Fig. 1), body weight changed rapidly in response to fluoxetine

104 administration.

105 Drug administration. Fluoxetine (TCI Chemicals, product \#F0750, Portland, OR, USA) was

106 initially dissolved in PBS at a concentration of $5 \mathrm{mg}$ per $\mathrm{ml}$. The resulting stock fluoxetine

107 solution, as well as the diluent PBS, was then frozen in separate aliquots at $-80^{\circ} \mathrm{C}$ to provide for

108 consistent solutions that were used for daily dosing. On the day of dosing, individual aliquots

109 were thawed and administered to mice by gavage (flexible plastic feeding needles \#9921,

110 Cadence Science, Cranston, RI, USA) to achieve a concentration of $20 \mathrm{mg}$ per kg body weight

111 with equal volumes of diluent administered to control animals. This dosage was selected

112 according to the landmark study of Dulawa et al. (Dulawa et al. 2004) which evaluated the dose

113 response relationship of chronic per oral fluoxetine administration in four strains of mice over an

114 approximately 24 day period in various models of anxiety and depression and determined that 18

$115 \mathrm{mg}$ per kg body weight was found to be active in all behavioral paradigms.

116 Behavioral procedures. At the conclusion of the 29-day dosing regimen, two behavioral

117 assessments, elevated-plus maze (EPM) and open field (OF), were conducted. These tests have

118 been well-characterized to measure anxiety-like behavior in mice (Prut \& Belzung 2003; Walf \&

119 Frye 2007). All movement and behavioral activity on the EPM and OF was digitally recorded 
120 using an HD 1080p webcam (Logitech, Newark, CA) coupled to a Windows-based computer

121 running the Any-maze behavioral tracking software (Stoelting Co., Wood Dale, IL, USA).

122 Statistical analyses of the behavioral data was performed using GraphPad Prism statistical

123 software package (version 7.05, GraphPad Software, La Jolla, CA, USA).

124 DNA isolation, MiSeq sequencing and sequence analyses. Immediately following the last

125 behavioral measure, mice were sacrificed via cardiac puncture. Fecal pellets were removed from

126 the large intestine and from the behavioral testing device immediately prior to sacrifice and

127 stored at $-80{ }^{\circ} \mathrm{C}$. Genomic DNA isolation was obtained using the PowerSoil DNA Isolation Kit

128 (MoBio, Carlsbad, CA).

129 In total, 60 fecal samples, representing 20 samples for each time point (day 0, 15, and 29)

130 with 10 samples for the control and treatment groups, respectively, were used for 16S rRNA

131 gene amplicon sequencing using Illumina MiSeq with $151 \mathrm{bp}$ paired-end sequencing technology.

132 16S rRNA gene PCR and library preparation and sequencing was completed at the

133 Environmental Sample Preparation and Sequencing Facility at Argonne National Laboratory. For

134 Illumina sequencing, Genomic DNA was amplified using the Earth Microbiome Project

135 barcoded primer set, adapted for the Illumina MiSeq by adding nine extra bases in the adapter

136 region of the forward amplification primer that support paired-end sequencing. The V4 region of

137 the 16S rRNA gene (515F-806R) was amplified with region-specific primers that included the

138 Illumina flowcell adapter sequences. The reverse amplification primer contained a twelve base

139 barcode sequence. Each $25 \mu \mathrm{l}$ PCR reaction contained $12 \mu \mathrm{l}$ of MoBio PCR Water (Certified

140 DNA-Free), $10 \mu \mathrm{l}$ of 5 Prime HotMasterMix (1x), $1 \mu \mathrm{l}$ of Forward Primer ( $5 \mu \mathrm{M}$ concentration,

$141200 \mathrm{pM}$ final), $1 \mu$ l Golay Barcode Tagged Reverse Primer (5 $\mu \mathrm{M}$ concentration, $200 \mathrm{pM}$ final),

142 and $1 \mu 1$ of template DNA. The conditions for PCR were as follows: $94^{\circ} \mathrm{C}$ for 3 minutes, with 35 
143 cycles at $94^{\circ} \mathrm{C}$ for $45 \mathrm{~s}, 50^{\circ} \mathrm{C}$ for $60 \mathrm{~s}$, and $72^{\circ} \mathrm{C}$ for $90 \mathrm{~s}$; with a final extension of $10 \mathrm{~min}$ at

$14472^{\circ} \mathrm{C}$. The PCR amplicons were quantified using PicoGreen (Invitrogen) and a plate reader.

145 Once quantified, different volumes of each of the products were pooled into a single tube so that

146 each amplicon is represented equally. This pool was then cleaned up using UltraClean ${ }^{\circledR}$ PCR

147 Clean-Up Kit (MoBIO), and then quantified using the Qubit (Invitrogen). After quantification,

148 the molarity of the pool was determined and diluted down to $2 \mathrm{nM}$, denatured, and then diluted

149 to a final concentration of $6.75 \mathrm{pM}$ with a $10 \%$ PhiX spike for sequencing on the Illumina

150 MiSeq. Sequence analysis was performed using mothur version 1.39.3 following the mothur

151 MiSeq SOP available at the mothur website (Kozich et al. 2013). Briefly, contigs were joined

152 with "make.contigs", reads longer than $250 \mathrm{bp}$, harboring any ambiguous bases or with more

153 than 8 consecutive homopolymers were excluded using "screen.seqs". Chimeric sequences were

154 removed with "chimera.uchime" and the reads were clustered into operational taxonomic units

155 (OTUs) using a 97\% similarity threshold and taxonomy was assigned to OTUs using the SILVA

156 NR 128 reference database (Quast et al. 2013). To identify biomarkers that differ in abundance

157 between groups were done with the LEfSe (Segata et al. 2011) implementation in mothur; for

158 this, p-values $<0.05$ were considered significant. As a first step, LEfSe performs a Kruskall-

159 Wallis test to analyze all features whether the values in different classes are differentially

160 distributed. In a second step, a pairwise Wilcoxon test is performed with the retained features. In

161 the last step, a linear discriminant analysis model is built from the retained features to determine

162 the effect sizes for each feature. For determination of differences between groups on community

163 level, analysis of molecular variance (AMOVA) and analysis of similarity (ANOSIM)

164 implemented in mothur was used. Heatmaps were generated with JColorGrid (Joachimiak et al.

165 2006). For better taxonomic classification, the OTUs were searched against the 16S rRNA gene 
166 sequences of the Mouse Intestinal Bacterial Collection isolates (miBC; (Lagkouvardos et al.

167 2016)) using BlastN.

168 Data availability

169 The sequencing data is available at the NCBI Sequence Read Archive SRA under accession 170 number SRP145610. 


\section{RESULTS}

172 As can be seen in Fig. 1, animals administered $20 \mathrm{mg} / \mathrm{kg}$ body weight of fluoxetine over a

173 29-day dosing period evidence changes in body weight while those administered diluent did not.

174 This dosage, as previously discussed in the Materials and Methods section, was chosen based

175 upon a landmark study examining the dose response relationship of chronic per oral

176 administration in three strains of mice and its ability to influence models of anxiety and

177 depression (Dulawa et al. 2004). In Figure 1A, the ability of fluoxetine to differentially increase

178 or decrease weight in select animals is evident while such dramatic shifts were not observed in

179 any of the control animals. As shown in Figure 1B, fluoxetine administration resulted in a

180 statistically significant ( $\mathrm{p}=0.018$, two-tailed t-test) change in weights gain/loss during the first 15

181 days. This result has also been observed in humans and animal models as administration of

182 fluoxetine can lead to dramatic changes in weight (Anderson 1998; Cascade et al. 2010).

183 Firmicutes were more abundant than Bacteroidetes in the fluoxetine treated mice (Fig. 2). As

184 such, there was no consistent change in one direction of weight gain or loss that could be correlated with the Firmicutes/Bacteroidetes ratios which has also been noted in humans (Sze \&

186 Schloss 2016). This observation of fluoxetine-induced changes in mice is also in agreement with

187 observations in the human population that it is a change in body weight, which could be either a 188 gain or loss, which is experienced in individuals administered given fluoxetine. Currently, there 189 are no predictive measures for the a priori prediction of the direction of the weight change.

190 As shown in Fig. 3, behavioral testing of mice tested at the end of the 29-day dosing 191 period revealed the development of mild anxiogenic-like behavior. For the EPM an increase in

192 entries into the closed arm (Fig. 3A; $p=0.047$ ) as well as decreased time in the center platform of 193 the EPM (Fig. 3B; $\mathrm{p}=0.030)$ were observed. For the OF an increase in total number of rearings 
194 in all zones (Fig. 3C; $p=0.008$ ), time rearing in the periphery zone (Fig. 3D; $p=0.009$ ), distance 195 traveled in the periphery (Fig. 3E; $p=0.042$ ) were observed while decreased time in the center 196 zone (Fig. 3F; $\mathrm{p}=0.035$ ) was seen. These fluoxetine anxiogenic-like induced effects are also 197 consistent with what has been observed in human and animal models (Aggarwal et al. 2016; 198 Anderson 1998; Cascade et al. 2010).

199 In total, 1.42 million reads were obtained after merging the forward and reverse reads.

200 After quality control, 1.134 million high quality reads remained which were clustered into 1,612

201 OTUs with at least 10 reads. On phylum level, the microbiota of the mice in this experiment was 202 dominated by Firmicutes (51.4\%), Bacteroidetes (44.8\%), Tenericutes (1.2\%), and

203 Deferibacteres (1.2\%) (Fig. 2). All other phyla showed less than 1\% relative abundance among 204 all samples.

205 The calculated species richness and diversity indicators were inconsistent and not 206 significantly different between the groups: Some animals showed an increase in species richness 207 and diversity during the trial, and others stayed similar or decreased (Table S1). No significant 208 differences ( $\mathrm{p}>0.57)$ were found in the richness and diversity estimators between the control and 209 the fluoxetine treated group. Most of the OTUs had highest similarity to phylotypes described as 210 members of the murine gastrointestinal tract (Fig. Z24, Tables S2, S3). Our results revealed clear 211 differences in abundance of OTUs between the two groups. Among all OTUs, 121 OTUs were 212 significantly different between control and the fluoxetine group; 21 of these OTUs were among 213 the 100 most abundant OTUs (Table S4). Some Bacteroidales S24-7 group, some

214 Lachnospiraceae OTUs and the Lactobacillus OTU17 (the latter showed a 7.4-fold decrease 215 under fluoxetine treatment) were significantly more abundant in the control group, whereas the 
216 Alistipes OTU24, the Lachnoclostridium and Anaerotruncus OTUs were more abundant in the

217 fluoxetine-treated mice.

218 On the genus level, Bacteroidales S24-7 group, unclassified Lachnospiraceae, and

219 Lachnospiraceae_NK4A136 group were most abundant (Fig. 5). Similar to our findings on OTU

220 level, a genus belonging to the Bacteroidales $S 24-7$ group and genera affiliating to the

221 Ruminococcaceae_UCG-014 group were significantly more abundant in the control group (Table

222 S5). Genera affiliating to the Lachnospiraceae_UCG-001 and UCG-006 groups, to

223 Anaerotruncus, Lachnoclostridium and to uncultured Lachnospiraceae showed significantly

224 higher abundance in the fluoxetine treated mice. In addition, also Anaerotruncus,

225 Ruminiclostridium_5, unclassified Coriobacteriaceae, and Lachnoclostridium were significantly

226 more abundant in the fluoxetine treated mice. Comparisons on whole community level using

227 AMOVA and ANOSIM revealed significant differences between the microbial communities of

228 the control group and the fluoxetine treated mice $(\mathrm{p}=0.02$ and $\mathrm{p}=0.05, \mathrm{R}=0.28$, respectively). 


\section{DISCUSSION}

230 Overall, our data revealed significant differences between the microbial communities of

231 mice from the control group compared to the fluoxetine treated mice indicative of a shift of

232 microbial communities towards dysbiosis induced by the fluoxetine treatment. As discussed in

233 prior sections, we chose to administer a chronic dose of fluoxetine (20 $\mathrm{mg}$ per $\mathrm{kg}$ of body

234 weight) that had been previously shown in a landmark study examining the dose response

235 relationship of chronic fluoxetine administration in four strains of mice to be the only one active

236 in all employed models of anxiety and depression (Dulawa et al. 2004). Specifically, a dose

237 response range from 0 to $25 \mathrm{mg}$ per $\mathrm{kg}$ body weight was employed with the finding that $18 \mathrm{mg}$

238 per kg was the only dosage found effective in all behavioral paradigms (Dulawa et al. 2004). As

239 can be seen in Figure 1 statistically significant changes in bodyweight occurred during the first

24015 days of administration that closely mimic what is observed in humans and other animal

241 models. Such dramatic shifts in weight gain/loss are often cited as a primary reason for patient

242 non-compliance in continuing drug therapy (Anderson 1998; Cascade et al. 2010). The ability of

243 this chronic dose of fluoxetine to influence standard measures of anxiety-like behavior in mice

244 was also observed (Figure 3) as has been noted in other studies which have employed chronic

245 administration of similar levels of per oral administered fluoxetine (Dulawa et al. 2004; Gosselin

246 et al. 2017).

247 The bioinformatic analyses examining the ability of fluoxetine to influence the

248 composition of the microbial communities demonstrated novel effects not previously reported in

249 the literature. Most strikingly, the analyses revealed that some OTUs were significantly higher

250 abundant in the control group, such as several Bacteroidales $S 24-7$ group OTUs, one

251 Lactobacillus OTU. Recently, an inhibition of growth of Lactobacillus by fluoxetine has been 
252 described (Cussotto et al. 2018) and Lactobacillus treatment reduced depressive-like behavior

253 (McVey Neufeld et al. 2018). It should be noted that not all Lactobacillus OTUs in our study

254 decreased due to the fluoxetine treatment. This could suggest that the fluoxetine treatment might

255 affect different Lactobacillus strains or species differently. This differential effect of fluoxetine

256 on lactobacilli is especially intriguing in light of the reports that demonstrate that specific species

257 and strains, such as L. reuteri (Marin et al. 2017) and L. rhamnosus JB-1 (McVey Neufeld et al.

258 2018) can influence behavior, while other lactobacilli cannot (Bravo et al. 2011; Vancanneyt et

259 al. 2006). A number of OTUs and genera were significantly more abundant in the treatment

260 group included Alistipes, various Lachnospiraceae OTUs, Lachnoclostridium and

261 Anaerotruncus. Alistipes belongs to the Bacteroidetes and is often found in murine

262 gastrointestinal tract microbiota samples (Lagkouvardos et al. 2016). Alistipes have recently been

263 found to be increased in abundance in patients with gastrointestinal complications after thoracic

264 aortic dissection surgery (Zheng et al. 2017), and associated with human colorectal carcinoma

265 (Shi et al. 2017). Alistipes have been shown to induce colitis and tumors in mice (Moschen et al.

266 2016) and were increased in mice exposed to ethanol (Peterson et al. 2017). Alistipes OTU24

267 might thus be an indicator of gastrointestinal dysbiosis. However, other Alistipes OTUs found in

268 this study (e.g. OTUs $23,25,47$ ) did not reveal significant differences in abundance between the

269 two experimental groups. A recent study has shown that Alistipes was reduced in abundance in

270 mice in response to chemically or pathogen induced colitis and this study indicated that Alistipes

271 is particularly sensitive to inflammation and possesses butyrate production capacity (Borton et al.

272 2017). This suggests that different phylotypes/OTUs within a genus or family can possess

273 different metabolic properties as suggested earlier (Berry et al. 2012). Anaerotruncus is a genus

274 in the Firmicutes, the function of its members remains largely unknown. Bacteria affiliating to 
275 the genus Anaerotruncus have been isolated from fecal samples from patients with obesity or 276 malnutrition (Pham et al. 2017; Togo et al. 2016) and have been associated with bacteremia in

277 humans (Lau et al. 2006). Furthermore, Anaerotruncus was increased in rats exposed to prenatal

278 stress (Golubeva et al. 2015). These data indicate that Anaerotruncus may be an indicator of 279 gastrointestinal dysbiosis. Several OTUs affiliating to the Lachnospiraceae family (OTUs 32, 38, 28086,93 ) were significantly more abundant in the fluoxetine treated mice. Our knowledge about 281 the metabolic properties of many members of the Lachnospiraceae is still limited. A recent study 282 has revealed members of unclassified Lachnospiraceae as important drivers of gastrointestinal 283 dysbiosis in mice (Moschen et al. 2016). differences between the two conditions. Two of these OTUs (45 and 74) were more abundant in the fluoxetine treated mice, whereas OTU69 was more abundant in the control group. In general,

287 Roseburia is considered a beneficial commensal bacterium producing butyrate and having a 288 positive impact on the host immune system and the abundance of Roseburia is decreasing under various disease conditions (Patterson et al. 2017; Tamanai-Shacoori et al. 2017). However, recently, a higher abundance of Roseburia was linked to gut dysbiois in mice and to cerebral in the fluoxetine treated mice provides preliminary evidence that drug-induced changes in microbial communities may be a contributing factor in the development of altered physiology. OTU46 is assigned to the genus Lachnoclostridium comprising members of the Clostridum cluster XIV including the former Clostridium scindens (Yutin \& Galperin 2013) and shows 99\% 16S rRNA gene similarity with C. scindens ATCC35704. Several C. scindens and

297 related Clostridum species are able to convert primary bile acids to toxic secondary bile acids 
298 such as deoxycholic acid and lithocholic acid which have been linked to diseases of the

299 gastrointestinal tract such as liver and colorectal cancer (Ridlon et al. 2016). We thus speculate

300 that the increase of OTU46 in the fluoxetine treated mice is a sign of dysbiosis, possibly by the

301 production of toxic secondary bile acids.

302

The most abundant OTUs and genus found in this study belong to the Bacteroidales S24-

3037 group, including the recently described Muribaculum intestinale (Lagkouvardos et al. 2016).

304 Members of the Bacteroidales group S24-7 are highly abundant particularly in murine and

305 human gastrointestinal tract samples (Borton et al. 2017; Lagkouvardos et al. 2016; Ormerod et

306 al. 2016). Both on OTU and genus level, Bacteroidales S24-7 showed higher abundance in the

307 control group samples. Recent evidence shows that members of the Bacteroidales S24-7 group

308 are able to produce propionate and have mucin degrading capacity (Borton et al. 2017; Ormerod

309 et al. 2016) suggesting a beneficial role for gastrointestinal tract health. In line with this, a

310 reduction of the Bacteroidales $\$ 24-7$ group has been described recently in colitic and obese mice

311 (Huazano-Garcia et al. 2017; Osaka et al. 2017). Furthermore, Bacteroidales S24-7 are reduced

312 in mice prone to develop dementia, suggesting a possible link of Bacteroidales S24-7 with

313 normal brain function (Sanguinetti et al. 2018).

314 The study by Cussotto et al. (Cussotto et al. 2018) showed that Prevotella and

315 Scuccinivibrio are decreased by fluoxetine. In our study, we did not identify Prevotella or

316 Succinivibrio among the 100 most abundant OTUs, which may be explained by different

317 experimental settings between the two studies such as different fluoxetine concentrations and

318 different animals (rat and mice).

319 The governing hypothesis in the present manuscript is based on the shared

320 neurochemistry between microbes and host, what has become known as microbial endocrinology 
321 (Lyte 2014). As shown, we have obtained the first data demonstrating that microbiota belonging

322 to a specific genera, such as Lactobacillus, and which have previously been shown to possess the

323 biogenic amine transporters PMAT and OCT (Lyte \& Brown 2018), can be influenced by

324 administration of an SSRI. It should however, be noted that the genes encoding the PMAT and

325 OCT transporters have not been identified yet. The ability of fluoxetine to affect the abundance

326 of the other genera discussed above raises the possibility that they may also possess biogenic

327 amine transporters similar to that of Lactobacilli. Our lab is currently examining this possibility,

328 but research is hampered by the fact that representative members of fluoxetine-affected genera in

329 this study are not available in microbial culture collections. The physiological role of PMAT-

330 and OCT-like transporter activity in Lactobacilli is not yet understood.

331 It is critical to separate the results, and implications, of the present study from that of

332 others in which direct antimicrobial effects of SSRIs were observed (Munoz-Bellido et al. 1996;

333 Munoz-Bellido et al. 2000). In those studies, antimicrobial effects were observed in an in vitro

334 assay system where there was no possibility for concentrations of fluoxetine that exceed what

335 can be achieved in vivo to be eliminated. Further, bacterial genera that were observed to be

336 susceptible to $\mathrm{mM}$ concentrations of fluoxetine, such as Escherichia, were not observed to be

337 decreased in the present study. As such, the working hypothesis is that fluoxetine is exerting its

338 effects through a PMAT- and OCT-like transporter in affected genera for which the precise

339 physiological role of this transporter has yet to be defined. The present data conclusively

340 demonstrates a genus-specific effect of fluoxetine that cannot be understood in the context of

341 solely antimicrobial activity for which concentrations needed to achieve an either bacteriostatic

342 or bactericidal effect need to be much higher than those that can be achieved in the gut. Given

343 the recent publication which has shown that in humans the administration of antipsychotics 
344 disrupts fecal microbial diversity (Maier et al. 2018), it is of importance to understand the

345 mechanisms by which such alterations occur and if they impact drug efficacy.

346 The role of the microbiota, and community structure, in determining behavior has come

347 under increasing investigation (Foster et al. 2016). A bi-directional axis involving the

348 microbiota, gut and brain has been proposed in which the microbiota influence behavior,

349 although mechanistic studies are still severely lacking. The need for identification of

350 mechanisms will be crucial if the concept of a microbiota-gut-brain axis is to progress especially

351 as regards eventual clinical application. The present study has shown that a microbial

352 endocrinology-based mechanism, namely on involving the PMAT- and OCT-like biogenic amine

353 transporters, may be one of the mechanisms responsible. The observed differential changes in

354 the abundance of specific microbiota genera that are well correlated (as has been discussed

355 above) with gut dysbiosis, suggests that the well-recognized negative side effects of fluoxetine

356 may be due to changes in gut microbiota that are mediated via a bacterial biogenic amine

357 transporter. The results of this study further suggest that restoration of physiologically beneficial

358 microbial diversity may have therapeutic potential to ameliorate some of the negative side effects

359 of fluoxetine thereby increasing drug efficacy and patient compliance. 


\section{CONCLUSIONS}

362

The present study has demonstrated that mice per orally administered fluoxetine exhibit

363 specific alterations in microbial community structure concomitant to alterations in body mass as

364 well as the development of mild anxiogenic-like behavior. As this study was undertaken to

365 examine the possible role of the microbiota as a contributing factor in the development of the

366 unwanted side effects with the use of fluoxetine in the treatment of anxiety-related illness in

367 humans, this report has provided first known initial evidence for the involvement of a specific

368 genus, Lactobacillus, that is also known to be involved in regulation of body mass. Further, as

369 prior work has shown that Lactobacilli possess a biogenic amine transporter capable of

370 incorporating fluoxetine (Lyte \& Brown 2018), it is proposed that a microbial endocrinology-

371 based specific mechanistic pathway is, in part, responsible for the fluoxetine-induced weight

372 alterations. By demonstrating the ability of fluoxetine to affect the microbial community

373 structure in a defined manner, the possibility that restoration of normal structure through the use

374 of probiotics may provide a means by which the negative clinical side effects of fluoxetine

375 leading to patient non-compliance in the treatment of depression may be ameliorated.

376

377 


\section{REFERENCES}

379 Aggarwal A, Jethani SL, Rohatgi RK, and Kalra J. 2016. Selective Serotonin Re-uptake Inhibitors (SSRIs) Induced Weight Changes: A Dose and Duration Dependent Study on Albino Rats.

382 J Clin Diagn Res 10:AF01-03. 10.7860/JCDR/2016/16482.7376

Anderson IM. 1998. SSRIS versus tricyclic antidepressants in depressed inpatients: a metaanalysis of efficacy and tolerability. Depression and Anxiety 7 Suppl 1:11-17.

Bagdy G, Graf M, Anheuer ZE, Modos EA, and Kantor S. 2001. Anxiety-like effects induced by acute fluoxetine, sertraline or $\mathrm{m}$-CPP treatment are reversed by pretreatment with the 5-HT2C receptor antagonist SB-242084 but not the 5-HT1A receptor antagonist WAY100635. International Journal of Neuropsychopharmacology 4:399-408. doi:10.1017/S1461145701002632

Berry D, Schwab C, Milinovich G, Reichert J, Ben Mahfoudh K, Decker T, Engel M, Hai B, Hainzl E, Heider S, Kenner L, Muller M, Rauch I, Strobl B, Wagner M, Schleper C, Urich T, and Loy A. 2012. Phylotype-level 16S rRNA analysis reveals new bacterial indicators of health state in acute murine colitis. ISME J 6:2091-2106. 10.1038/ismej.2012.39

Borton MA, Sabag-Daigle A, Wu J, Solden LM, O'Banion BS, Daly RA, Wolfe RA, Gonzalez JF, Wysocki VH, Ahmer BMM, and Wrighton KC. 2017. Chemical and pathogen-induced inflammation disrupt the murine intestinal microbiome. Microbiome 5:47. $10.1186 / s 40168-017-0264-8$

Bravo JA, Forsythe P, Chew MV, Escaravage E, Savignac HM, Dinan TG, Bienenstock J, and Cryan JF. 2011. Ingestion of Lactobacillus strain regulates emotional behavior and central GABA receptor expression in a mouse via the vagus nerve. Proceedings of the National 
400

401

402

403

404

405

406

407

408

409

410

411

412

413

414

415

416

417

418

419

420

Academy of Sciences of the United States of America 108:16050-16055.

10.1073/pnas.1102999108

Cascade E, Kalali AH, Mehra S, and Meyer JM. 2010. Real-world Data on Atypical Antipsychotic Medication Side Effects. Psychiatry (Edgmont) 7:9-12.

Cussotto S, Strain CR, Fouhy F, Strain RG, Peterson VL, Clarke G, Stanton C, Dinan TG, and Cryan JF. 2018. Differential effects of psychotropic drugs on microbiome composition and gastrointestinal function. Psychopharmacology. 10.1007/s00213-018-5006-5

Dulawa SC, Holick KA, Gundersen B, and Hen R. 2004. Effects of chronic fluoxetine in animal models of anxiety and depression. Neuropsychopharmacology 29:1321-1330. 10.1038/sj.npp.1300433

Foster JA, Lyte M, Meyer E, and Cryan JF. 2016. Gut Microbiota and Brain Function: An Evolving Field in Neuroscience. International Journal of Neuropsychopharmacology 19. 10.1093/ijnp/pyv114

Golubeva AV, Crampton S, Desbonnet L, Edge D, O'Sullivan O, Lomasney KW, Zhdanov AV, Crispie F, Moloney RD, Borre YE, Cotter PD, Hyland NP, O'Halloran KD, Dinan TG, O'Keeffe GW, and Cryan JF. 2015. Prenatal stress-induced alterations in major physiological systems correlate with gut microbiota composition in adulthood. Psychoneuroendocrinology 60:58-74. 10.1016/j.psyneuen.2015.06.002

Gosselin T, Le Guisquet AM, Brizard B, Hommet C, Minier F, and Belzung C. 2017. Fluoxetine induces paradoxical effects in C57BL6/J mice: comparison with BALB/c mice. Behavioural Pharmacology 28:466-476. 10.1097/FBP.0000000000000321 
421 Huazano-Garcia A, Shin H, and Lopez MG. 2017. Modulation of Gut Microbiota of Overweight

$422 \quad$ Mice by Agavins and Their Association with Body Weight Loss. Nutrients 9.

423 $10.3390 /$ nu9090821

424 Joachimiak MP, Weisman JL, and May B. 2006. JColorGrid: software for the visualization of 425 biological measurements. BMC Bioinformatics 7:225. 10.1186/1471-2105-7-225

426

427

428

429

430

431

432

433

434

435

436

437

438

439

440

441

442

Kozich JJ, Westcott SL, Baxter NT, Highlander SK, and Schloss PD. 2013. Development of a dualindex sequencing strategy and curation pipeline for analyzing amplicon sequence data on the MiSeq Illumina sequencing platform. Applied and Environmental Microbiology 79:5112-5120. 10.1128/AEM.01043-13

Lagkouvardos I, Pukall R, Abt B, Foesel BU, Meier-Kolthoff JP, Kumar N, Bresciani A, Martinez I, Just S, Ziegler C, Brugiroux S, Garzetti D, Wenning M, Bui TP, Wang J, Hugenholtz F, Plugge CM, Peterson DA, Hornef MW, Baines JF, Smidt H, Walter J, Kristiansen K, Nielsen HB, Haller D, Overmann J, Stecher B, and Clavel T. 2016. The Mouse Intestinal Bacterial Collection (miBC) provides host-specific insight into cultured diversity and functional potential of the gut microbiota. Nat Microbiol 1:16131. 10.1038/nmicrobiol.2016.131

Lau SK, Woo PC, Woo GK, Fung AM, Ngan AH, Song Y, Liu C, Summanen P, Finegold SM, and Yuen K. 2006. Bacteraemia caused by Anaerotruncus colihominis and emended description of the species. Journal of Clinical Pathology 59:748-752. 10.1136/jcp.2005.031773

Lemberger L, Bergstrom RF, Wolen RL, Farid NA, Enas GG, and Aronoff GR. 1985. Fluoxetine: clinical pharmacology and physiologic disposition. Journal of Clinical Psychiatry 46:1419. 
443 Lyte M. 2014. Microbial endocrinology and the microbiota-gut-brain axis. Advances in

444

445

446

447

448

449

450

451

452

453

454

455

456

457

458

459

460

461

462

463

Experimental Medicine and Biology 817:3-24. 10.1007/978-1-4939-0897-4_1

Lyte M, and Brown DR. 2018. Evidence for PMAT- and OCT-like biogenic amine transporters in a probiotic strain of Lactobacillus: Implications for interkingdom communication within the microbiota-gut-brain axis. PloS One 13:e0191037. 10.1371/journal.pone.0191037

Maier L, Pruteanu M, Kuhn M, Zeller G, Telzerow A, Anderson EE, Brochado AR, Fernandez KC, Dose $\mathrm{H}$, Mori H, Patil KR, Bork P, and Typas A. 2018. Extensive impact of non-antibiotic drugs on human gut bacteria. Nature 555:623-628. 10.1038/nature25979

Marin IA, Goertz JE, Ren T, Rich SS, Onengut-Gumuscu S, Farber E, Wu M, Overall CC, Kipnis J, and Gaultier A. 2017. Microbiota alteration is associated with the development of stress-induced despair behavior. Scientific Reports 7:43859. 10.1038/srep43859

McCafferty J, Muhlbauer M, Gharaibeh RZ, Arthur JC, Perez-Chanona E, Sha W, Jobin C, and Fodor AA. 2013. Stochastic changes over time and not founder effects drive cage effects in microbial community assembly in a mouse model. ISME J 7:2116-2125. 10.1038/ismej.2013.106

McVey Neufeld KA, Kay S, and Bienenstock J. 2018. Mouse Strain Affects Behavioral and Neuroendocrine Stress Responses Following Administration of Probiotic Lactobacillus rhamnosus JB-1 or Traditional Antidepressant Fluoxetine. Frontiers in Neuroscience 12:294. 10.3389/fnins.2018.00294

Moschen AR, Gerner RR, Wang J, Klepsch V, Adolph TE, Reider SJ, Hackl H, Pfister A, Schilling J, Moser PL, Kempster SL, Swidsinski A, Orth Holler D, Weiss G, Baines JF, Kaser A, and Tilg 
464

465

466

467

468

469

470

471

472

473

474

475

476

477

478

479

480

481

482

483

484

H. 2016. Lipocalin 2 Protects from Inflammation and Tumorigenesis Associated with Gut Microbiota Alterations. Cell Host \& Microbe 19:455-469. 10.1016/j.chom.2016.03.007

Munoz-Bellido JL, Munoz-Criado S, and Garcia-Rodriguez JA. 1996. In-vitro activity of psychiatric drugs against Corynebacterium urealyticum (Corynebacterium group D2). Journal of Antimicrobial Chemotherapy 37:1005-1009.

Munoz-Bellido JL, Munoz-Criado S, and Garcia-Rodriguez JA. 2000. Antimicrobial activity of psychotropic drugs: selective serotonin reuptake inhibitors. International Journal of Antimicrobial Agents 14:177-180.

Ormerod KL, Wood DL, Lachner N, Gellatly SL, Daly JN, Parsons JD, Dal'Molin CG, Palfreyman RW, Nielsen LK, Cooper MA, Morrison M, Hansbro PM, and Hugenholtz P. 2016. Genomic characterization of the uncultured Bacteroidales family S24-7 inhabiting the guts of homeothermic animals. Microbiome 4:36. 10.1186/s40168-016-0181-2

Osaka T, Moriyama E, Arai S, Date Y, Yagi J, Kikuchi J, and Tsuneda S. 2017. Meta-Analysis of Fecal Microbiota and Metabolites in Experimental Colitic Mice during the Inflammatory and Healing Phases. Nutrients 9. 10.3390/nu9121329

Patterson AM, Mulder IE, Travis AJ, Lan A, Cerf-Bensussan N, Gaboriau-Routhiau V, Garden K, Logan E, Delday MI, Coutts AGP, Monnais E, Ferraria VC, Inoue R, Grant G, and Aminov RI. 2017. Human Gut Symbiont Roseburia hominis Promotes and Regulates Innate Immunity. Frontiers in Immunology 8:1166. 10.3389/fimmu.2017.01166

Peterson VL, Jury NJ, Cabrera-Rubio R, Draper LA, Crispie F, Cotter PD, Dinan TG, Holmes A, and Cryan JF. 2017. Drunk bugs: Chronic vapour alcohol exposure induces marked changes in 
the gut microbiome in mice. Behavioural Brain Research 323:172-176.

486 10.1016/j.bbr.2017.01.049

487

Pham TP, Cadoret F, Alou MT, Brah S, Diallo BA, Diallo A, Sokhna C, Delerce J, Fournier PE, Million M, and Raoult D. 2017. 'Urmitella timonensis' gen. nov., sp. nov., 'Blautia marasmi' sp. nov., 'Lachnoclostridium pacaense' sp. nov., 'Bacillus marasmi' sp. nov. and 'Anaerotruncus rubiinfantis' sp. nov., isolated from stool samples of undernourished African children. New Microbes New Infect 17:84-88. 10.1016/j.nmni.2017.02.004

Prut L, and Belzung C. 2003. The open field as a paradigm to measure the effects of drugs on anxiety-like behaviors: a review. European Journal of Pharmacology 463:3-33.

Quast C, Pruesse E, Yilmaz P, Gerken J, Schweer T, Yarza P, Peplies J, and Glockner FO. 2013. The SILVA ribosomal RNA gene database project: improved data processing and webbased tools. Nucleic Acids Research 41:D590-596. 10.1093/nar/gks1219

Ridlon JM, Harris SC, Bhowmik S, Kang DJ, and Hylemon PB. 2016. Consequences of bile salt biotransformations by intestinal bacteria. Gut Microbes 7:22-39. $10.1080 / 19490976.2015 .1127483$

Sanguinetti E, Collado MC, Marrachelli VG, Monleon D, Selma-Royo M, Pardo-Tendero MM, Burchielli S, and lozzo P. 2018. Microbiome-metabolome signatures in mice genetically prone to develop dementia, fed a normal or fatty diet. Scientific Reports 8:4907.

$$
10.1038 / s 41598-018-23261-1
$$

Segata N, Izard J, Waldron L, Gevers D, Miropolsky L, Garrett WS, and Huttenhower C. 2011. Metagenomic biomarker discovery and explanation. Genome Biology 12:R60. 10.1186/gb-2011-12-6-r60 
507 Shi X, Chang C, Ma S, Cheng Y, Zhang J, and Gao Q. 2017. Efficient bioconversion of L-glutamate 508 to gamma-aminobutyric acid by Lactobacillus brevis resting cells. Journal of Industrial $509 \quad$ Microbiology \& Biotechnology 44:697-704. 10.1007/s10295-016-1777-z

510 Sze MA, and Schloss PD. 2016. Looking for a Signal in the Noise: Revisiting Obesity and the $511 \quad$ Microbiome. MBio 7. 10.1128/mBio.01018-16

512 Tamanai-Shacoori Z, Smida I, Bousarghin L, Loreal O, Meuric V, Fong SB, Bonnaure-Mallet M,

513

514

515

516

517

518

519

520

521

522

523

524

525

526 and Jolivet-Gougeon A. 2017. Roseburia spp.: a marker of health? Future Microbiology 12:157-170. 10.2217/fmb-2016-0130

Togo AH, Valero R, Delerce J, Raoult D, and Million M. 2016. "Anaerotruncus massiliensis," a new species identified from human stool from an obese patient after bariatric surgery. New Microbes New Infect 14:56-57. 10.1016/j.nmni.2016.07.015

Vancanneyt M, Huys G, Lefebvre K, Vankerckhoven V, Goossens H, and Swings J. 2006. Intraspecific genotypic characterization of Lactobacillus rhamnosus strains intended for probiotic use and isolates of human origin. Applied and Environmental Microbiology 72:5376-5383. 10.1128/AEM.00091-06

Walf AA, and Frye CA. 2007. The use of the elevated plus maze as an assay of anxiety-related behavior in rodents. Nature Protocols 2:322-328. 10.1038/nprot.2007.44

Williams T, Hattingh CJ, Kariuki CM, Tromp SA, van Balkom AJ, Ipser JC, and Stein DJ. 2017. Pharmacotherapy for social anxiety disorder (SAnD). Cochrane Database of Systematic Reviews 10:CD001206. 10.1002/14651858.CD001206.pub3 
527 Yutin N, and Galperin MY. 2013. A genomic update on clostridial phylogeny: Gram-negative 528 spore formers and other misplaced clostridia. Environmental Microbiology 15:2631-

529 2641. 10.1111/1462-2920.12173

530 Zheng S, S. S, Qiao Z, Chen X, Piao C, Yu Y, Gao F, Zhang J, and Du J. 2017. Clinical Parameters

531 and Gut Microbiome Changes Before and After Surgery in Thoracic Aortic Dissection in

$532 \quad$ Patients with Gastrointestinal Complications. Sci Rep 7:15228. 10.1038/s41598-017-

$533 \quad 15079-0$

534

535

536 
537 Fig. 1: (A) Weight changes over time in control and fluoxetine-dosed mice ( $\mathrm{N}=10$ per group).

538 (B) Percent changes in bodyweight over time in control and fluoxetine-dosed animals ( $\mathrm{N}=10$ per

539 group). Statistical analyses were conducted and $\mathrm{p}$ values are shown in the figure (two-tailed $\mathrm{t}$

540 tests).

541 Fig. 2: Relative abundance of bacterial phyla. The figure shows mean relative abundance values

542 for Firmicutes, Bacteroidetes, Tenericutes, and Proteobacteria in the control group and

543 fluoxetine-treated mice. Error bars represent standard error of the mean.

544 Fig. 3: Behavioral assessment of control ( $N=10$ per group) and fluoxetine-dosed $(\mathrm{N}=10$ per

545 group) mice at conclusion of 29-day dosing regimen. A. Entries into closed arm of EPM; B.

546 Time in center platform of the EPM; C. Total rearings in all zones of the OF; D. Time rearing in

547 the periphery zone of the OF; E. Distance traveled in the periphery zone of the OF; and F. Time

548 in the center zone of the OF. Statistical analyses were conducted and $p$ values are shown in the

549 figure (two-tailed t tests).

550 Fig. 4: Relative abundance of the 30 most abundant OTUs. The heatmap shows median relative

551 abundance values for OTUs in the control group and fluoxetine-treated mice. OTUs which were

552 statistically significantly different between the two groups based on LEfSe (Segata et al. 2011)

553 are highlighted in bold and by asterisks (see Table S4 for details).

554 Fig. 5: Relative abundance of the 20 most abundant genera. The heatmap shows median relative 555 abundance values for genera in the control group and fluoxetine-treated mice. Genera which

556 were statistically significantly different between the two groups based on LEfSe (Segata et al.

557 2011) are highlighted in bold and by asterisks (See Table S5 for details).

558 
Figure 1

(A) Weight changes over time in control and fluoxetine-dosed mice ( $\mathrm{N}=10$ per group) and $(B)$ Percent changes in bodyweight over time in control and fluoxetine-dosed animals ( $\mathrm{N}=10$ per group)

Statistical analyses were conducted and $p$ values are shown in the figure (two-tailed t tests).

A

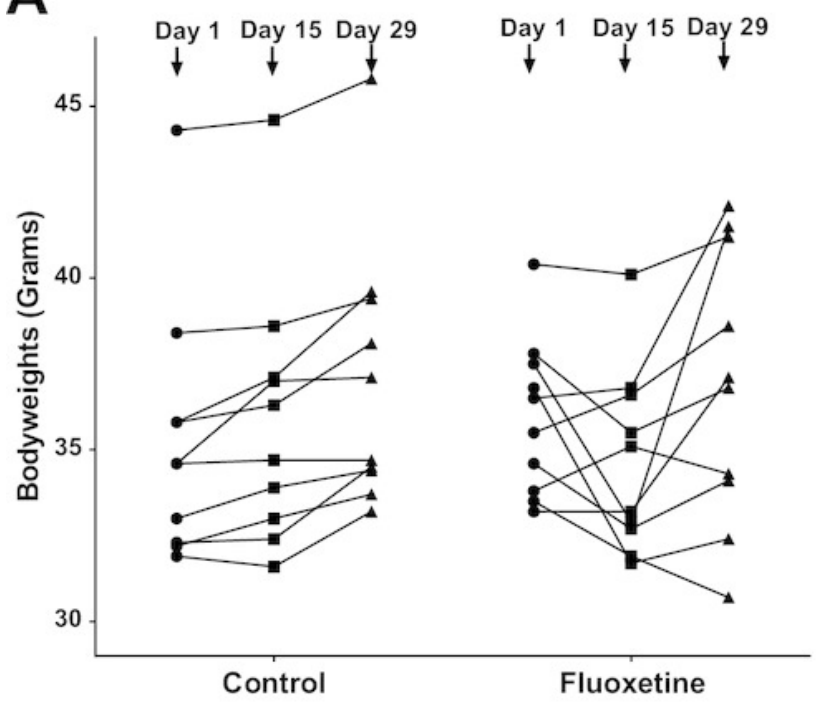

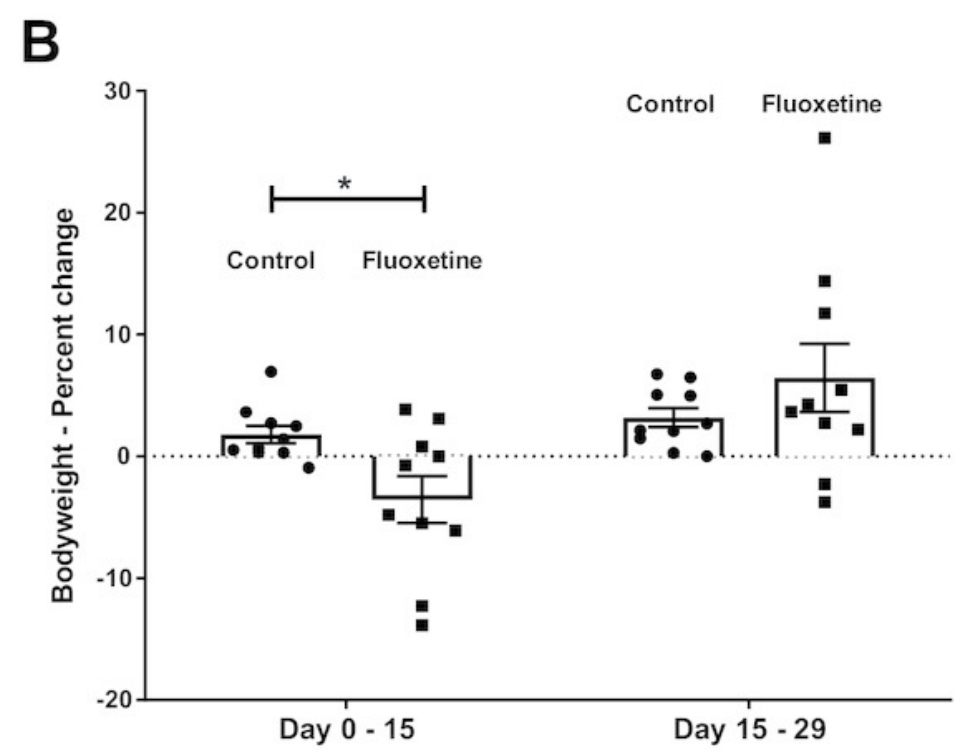




\section{Figure 2}

Relative abundance of bacterial phyla

The figure shows mean relative abundance values for Firmicutes, Bacteroidetes, Tenericutes, and Proteobacteria in the control group and fluoxetine-treated mice. Error bars represent standard error of the mean.

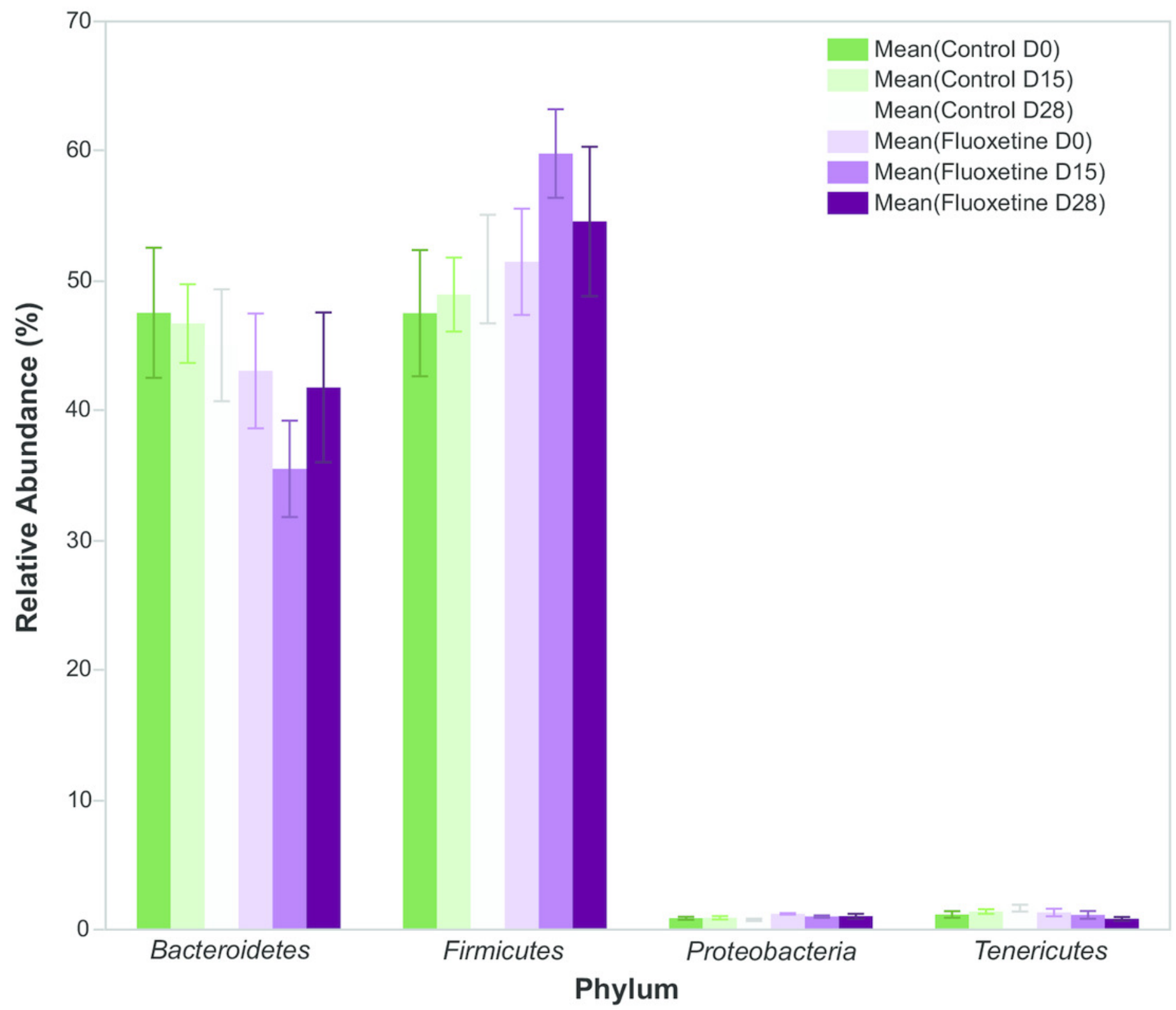


Figure 3

Behavioral assessment of control ( $\mathrm{N}=10$ per group) and fluoxetine-dosed ( $\mathrm{N}=10$ per group) mice at conclusion of 29-day dosing regimen

A. Entries into closed arm of EPM; B. Time in center platform of the EPM; C. Total rearings in all zones of the OF; D. Time rearing in the periphery zone of the OF; E. Distance traveled in the periphery zone of the OF; and F. Time in the center zone of the OF. Statistical analyses were conducted and $\mathrm{p}$ values are shown in the figure (two-tailed t tests).
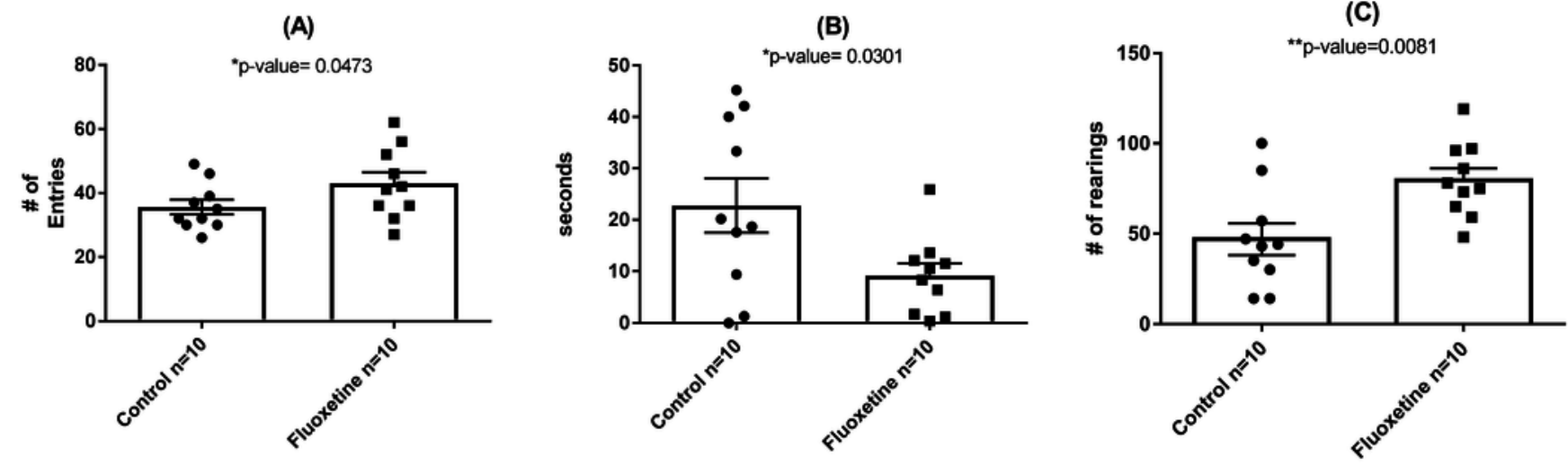

(D)
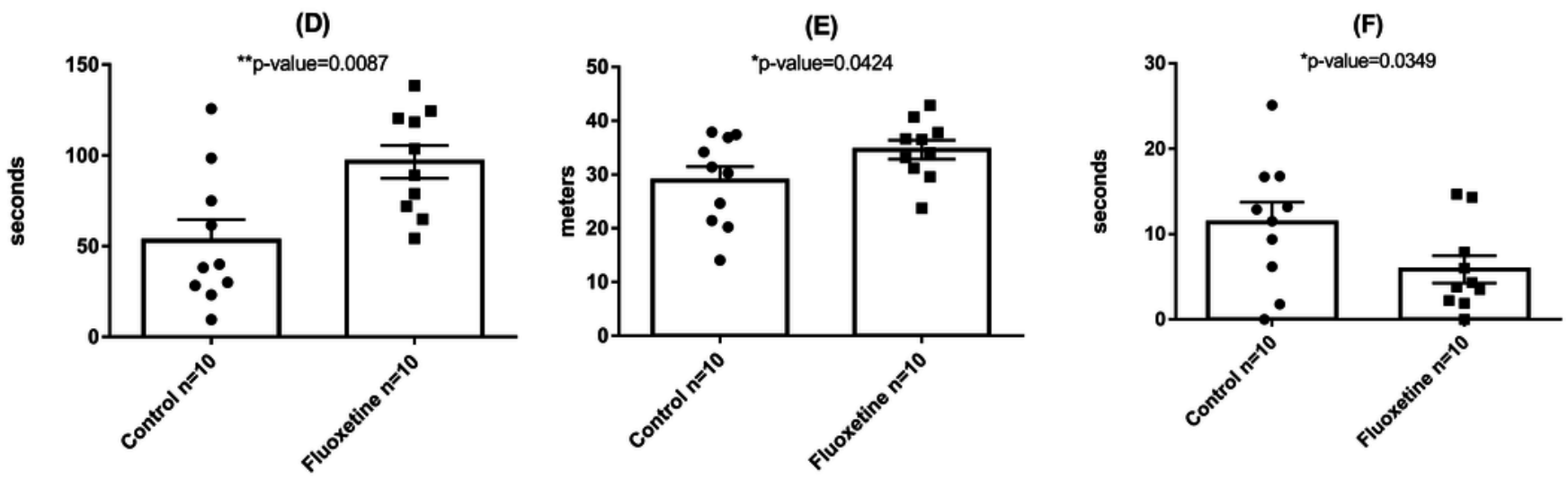


\section{Figure 4}

Relative abundance of the 30 most abundant OTUs

The heatmap shows median relative abundance values for OTUs in the control group and fluoxetine-treated mice. OTUs which were statistically significantly different between the two groups based on LEfSe (Segata et al. 2011) are highlighted in bold and by asterisks (see Table S4 for details). 
OTU1 Rikenellaceae_RC_gut_group

OTU2 Bacteroidales_s24-7_group

OTU3 Bacteroidales_S24-7_group

OTU4 Bacteroidales_S24-7_group

OTU5 Lactobacillus apodemi

OTU6 Bacteroidales_S24-7_group, $\mathrm{p}=0.01$

OTU7 Bacteroides acidifaciens

OTU8 Bacteroidales_S24-7_group

OTU9 Roseburia faecis

OTU10 Lachnospiraceae_UCG-001

OTU11 Lachnospiraceae_NK4A136_group

OTU12 Bacteroides acidifaciens

OTU13 Bacteroidales_S24-7_group

OTU14 Lachnospiraceae_UCG-001

OTU15 Bacteroidales_S24-7_group

OTU16 Lachnospiraceae_NK4A136_group

OTU17 Lactobacillus johnsonii, p=0.01

OTU18 Mucispirillum schaedleri

OTU19 Bacteroidales_S24-7_group, $\mathrm{p}=0.02$

OTU20 lleibacterium valens

OTU21 Lachnospiraceae_NK4A136_group

OTU22 Bacteroidales_S24-7_group

OTU23 Alistipes finegoldii

OTU24 Alistipes finegoldii, $\mathrm{p}=0.02$

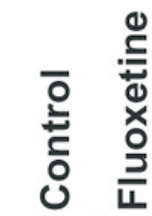

Relative abundance (\%)
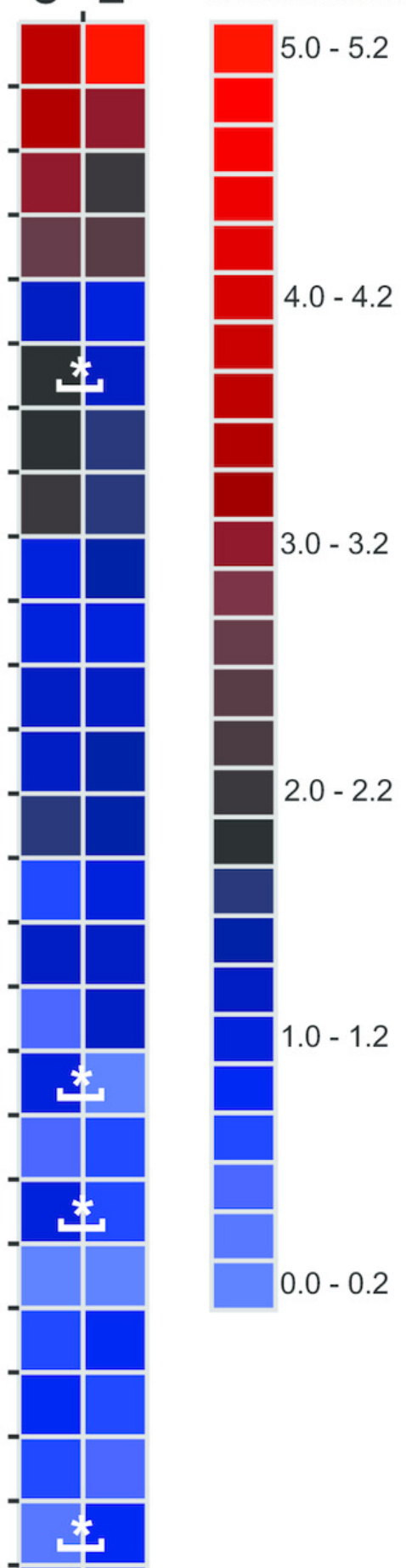

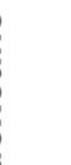

OTU25 Alistipes onderdonkii

OTU26 Rikenellaceae_unclassified

OTU27 Lactobacillus reuteri

OTU28 Lachnospiraceae_unclassified, p=0.02 


\section{Figure 5}

Relative abundance of the 20 most abundant genera

The heatmap shows median relative abundance values for genera in the control group and fluoxetine-treated mice. Genera which were statistically significantly different between the two groups based on LEfSe (Segata et al. 2011) are highlighted in bold and by asterisks (See Table S5 for details). 
Bacteroidales_S24-7_group, $\mathrm{p}=\mathbf{0 . 0 3}$

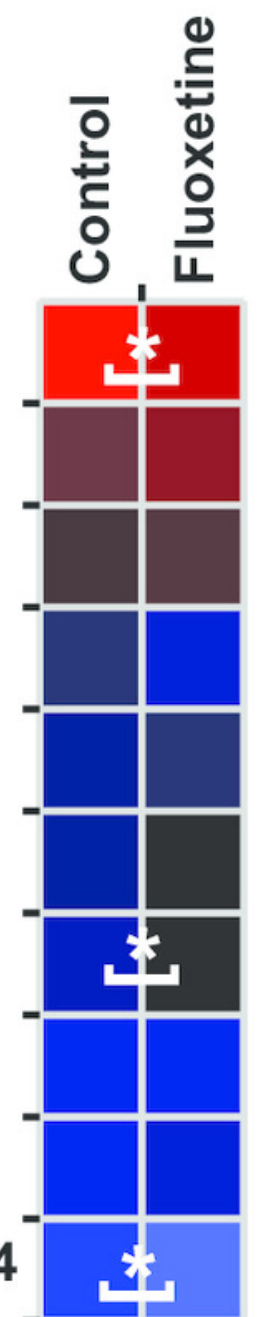

Relative abundance (\%)

Lachnospiraceae_unclassified

Lachnospiraceae_NK4A136_group

Lactobacillus

Bacteroides

Rikenellaceae_RC9_gut_group

Lachnospiraceae_UCG-001, p=0.04

Alistipes

Roseburia

Ruminococcaceae_UCG-014, p=0.004

Ruminococcaceae_unclassified

Mollicutes_RF9, $\mathrm{p}=0.01$

Ruminiclostridium_9

Ruminiclostridium

Clostridiales_vadinBB60_group

Lachnospiraceae_uncultured, $\mathrm{p}=0.01$

Rikenellaceae_unclassified

Mucispirillum

Lachnoclostridium, $\mathrm{p}=\mathbf{0 . 0 0 4}$

Bacteria_unclassified

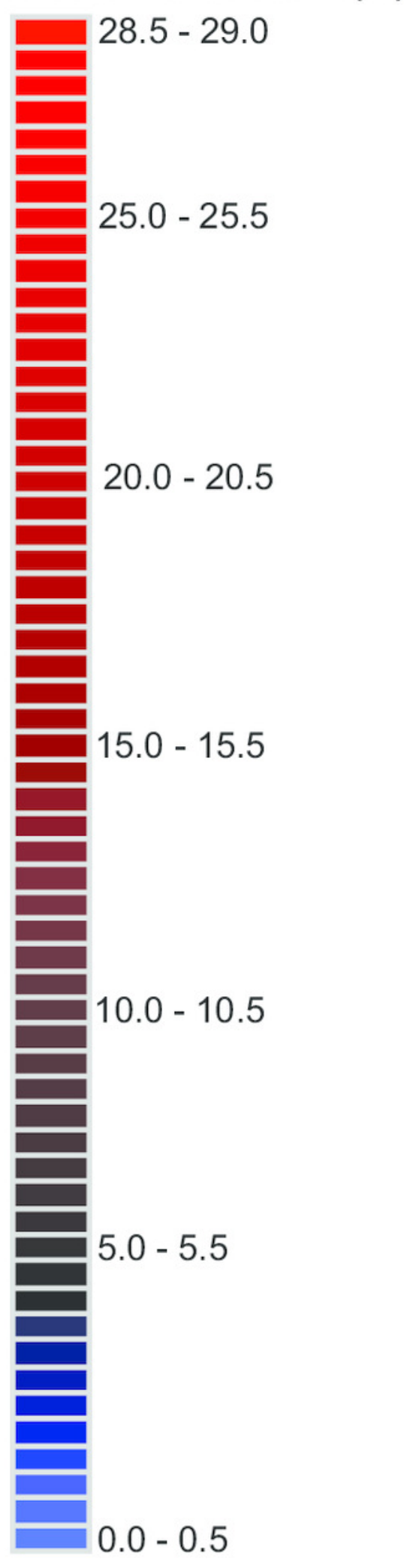

